\title{
Factores del riesgo en la cartera de microcréditos de los bancos universales
}

\author{
Risk factors in the universal bank's microcredit portfolio
}

\section{Fatores de risco na carteira de microcrédito do universal banks}

Recibido: enero 2020

Arbitrado: febrero 2020

Publicado: mayo 2020
ABSTRACT

One of the great components of the economy is credit, a financial tool that can be used to make investments or meet needs. From this perspective, this article seeks to determine the risk factors in the microcredit portfolio of universal banks. The methodology was descriptive, with a non-experimental, transectional field design. The population was made up of (8) eight universal banks of the Cabimas municipality. For data collection, the survey was used through a questionnaire with a frequency scale. Validity was performed through expert judgment, and its reliability was 0.92 calculated by Cronbach's Alpha Coefficient. Data analysis was performed with descriptive statistics, based on the arithmetic mean. The organizational structure, legal risk, economy and economic activity of the client were determined as factors that give strengths to the analyzed banks.

Key words: Universal banks; microcredit portfolio; credit; risk factors, financial tool
RESUMO
《 Marlin Ocando

mrlinocando@gmail.com

ORCID: 0000-0001-8412-4198

sta Oriental del Lago, Venezuela economía es el crédito, herramienta financiera inversiones o cubrir necesidades. Desde esta diseño de campo no experimental transeccional. La población quedó conformada por (8) ochos bancos universales del municipio Cabimas. Para la recolección de se empleó la encuesta mediante cuestionario con escala de frecuencias. se realizó a través del juicio calculada por el Coeficiente Alfa de Cronbach. El análisis de los datos se realizó con estadística descriptiva, basada en la media aritmética. Se determinaron como factores que le otorgan fortalezas a los bancos analizados la estructura organizacional, el riesgo legal, la economía y la actividad económica del cliente.

Palabras clave: Bancos universales; cartera de microcréditos; crédito; factores del riesgo, herramienta financiera
Um dos grandes componentes da economia é o crédito, uma ferramenta financeira que pode ser usada para fazer investimentos ou atender necessidades. Nessa perspectiva, este artigo busca determinar os fatores de risco na carteira de microcrédito dos bancos universais. A metodologia foi descritiva, com um delineamento de campo transversal não experimental. A população era composta por (8) oito bancos universais do município de Cabimas. Para a coleta de dados, utilizou-se a pesquisa por meio de um questionário com escala de frequência. A validade foi realizada por meio de julgamento de especialistas e sua confiabilidade foi de 0,92 calculada pelo coeficiente alfa de Cronbach. A análise dos dados foi realizada com estatística descritiva, com base na média aritmética. A estrutura organizacional, o risco jurídico, a economia e a atividade económica do cliente foram determinados como fatores que dão força aos bancos analisados.

Palavras-chave: Bancos universais; carteira de microcrédito; crédito; fatores de risco, ferramenta financeira 


\section{INTRODUCCIÓN}

A dministrar recursos financieros es el negocio cotidiano de los bancos; no obstante la globalización financiera ha venido generando una mayor volatilidad de las variables macroeconómicas que ocasiona la complejidad de la supervisión de las instituciones financieras y la seguridad del sistema financiero y ha repercutido de forma directa en la búsqueda de una adecuada administración del riesgo en el interior de las propias instituciones financieras. De acuerdo con Gootkind (2013), el riesgo crediticio es el riesgo de pérdida como resultado de que el prestatario (emisor de deuda) no pueda hacer los pagos completos y oportunos de intereses y/o principal. El riesgo de crédito tiene dos componentes. El primero se conoce como riesgo de impago, o la probabilidad de incumplimiento, es decir, la posibilidad de que un prestatario no pague, incumpliendo con su obligación de hacer los pagos completos y oportunos de principal e intereses, de acuerdo con los términos del contrato de la deuda. El segundo componente es la severidad de la pérdida en caso de incumplimiento, es decir, la parte del valor de un bono (incluyendo intereses no pagados) que el inversionista pierde. Un incumplimiento puede dar lugar a pérdidas de diversas magnitudes.

Así, para toda entidad dentro del sistema financiero, es importante definir los factores relacionados al riesgo de crédito mediante herramientas y parámetros, pues, de estos factores volátiles y sensibles al entorno macroeconómico que lo rodea, se encuentra la clave para poder enfrentar de la mejor manera la incertidumbre que depara el futuro del negocio, lo que favorecería de forma ventajosa el posicionamiento de la entidad ante sus competidores.

En este contexto, están inmersos los bancos universales del municipio Cabimas, los cuales según el diagnóstico, están mostrando claras señales de desaceleración en la expansión y crecimiento en la cartera de microcréditos a causa de los requerimientos mínimos de solvencia exigidos, y las limitaciones por regulaciones en los diversos ingresos por su gestión natural. Partiendo de lo antes expuesto, se realizó una investigación descriptiva, con el propósito de determinar los factores del riesgo en la cartera de microcréditos de los bancos universales.

\section{MÉTODO}

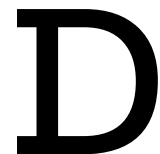

e acuerdo con el objetivo planteado, la metodología fue de tipo descriptiva, debido a que su propósito se centró en determinar los factores del riesgo en la cartera de microcréditos de los bancos universales. Al respecto, Tamayo (2009) expone que la investigación descriptiva trabaja sobre realidades de hecho y su característica esencial es presentar una interpretación correcta. 
Una vez establecido el tipo de investigación se plantea el diseño de la misma, el cual se refiere al plan o estrategia que se desarrollará para obtener la información, analizar los datos que permitan dar respuesta a las preguntas formuladas y alcanzar los objetivos o intereses trazados. En este sentido, este estudio se ubica como una investigación de campo, no experimental, transeccional, debido a que los factores del riesgo, se describieron tal y como se presentaba en los bancos universales del municipio Cabimas, cuyos sujetos informantes fueron 17 analistas y 12 gerentes.

Para la recolección de la información se aplicó como técnica la encuesta y como instrumento un cuestionario, con escala de frecuencia. La validación del mismo se realizó mediante el juicio de cinco (5) expertos en el área y su confiabilidad se calculó a través del Coeficiente Alfa de Cronbach, donde se obtuvo como valor de confiabilidad total del instrumento 0,92. Una vez recogida la información, el análisis estadístico de los datos se realizó a través de la estadística descriptiva, que permitió caracterizar cada una de factores del riesgo, a través del uso de la media aritmética, posterior a ello se utilizó la estadística inferencial aplicando un análisis de correlación regresión lineal.

\section{RESULTADOS Y DISCUSIÓN}

$\mathrm{L}$ a tabla 1 muestra los resultados para la dimensión factores internos donde se resumen los hallazgos en políticas $(2,75)$ riesgo operacional $(2,42)$ estructura organizacional $(4,25)$ y riesgo legal $(3,83)$ como indicadores del comportamiento de esta dimensión refiriendo su moderada presencia $(3,31)$ en los bancos universales del municipio Cabimas, Venezuela.

Esto ratifica lo planteado por Soley y Rahnema (2006) señalando que la clasificación de las exposiciones a los riesgos dependerá de las propias estimaciones internas de los riesgos que realice el banco. Si el banco posee un sistema de gestión de riesgos que permite calcular las probabilidades de impago de sus prestatarios, y que haya estado en vigor durante los tres últimos años y ha sido reconocida su validez por el supervisor, podrá usar estas probabilidades de impago para clasificar los préstamos en categorías, y con ello obtener las ponderaciones que servirán para calcular los requerimientos de capital.

Tabla 1. Factores internos.

\begin{tabular}{lcl}
\hline \multicolumn{1}{c}{ Indicadores } & Media & \multicolumn{1}{c}{ Categoría } \\
\hline Políticas (P) & 2,75 & Moderada presencia/ Leve fortaleza \\
Riesgo operacional (RO) & 2,42 & Baja presencia/ Debilidad \\
Estructura organizacional (EO) & 4,25 & Muy alta presencia/ Fortaleza \\
Riesgo legal (RL) & 3,83 & Alta presencia/ Fortaleza \\
\hline \multicolumn{1}{c}{ Dimensión } & 3,31 & Moderada presencia/ leve fortaleza \\
\hline
\end{tabular}


Dado estos resultados, se visualiza los factores internos con respecto al riesgo crediticio de los bancos universales como leves fortalezas que pudiese favorecer su desarrollo financiero, sin embargo debe ser mejorado, a fin de que la banca pueda lograr una buena gestión en materia de recursos financieros, en tal sentido el solicitante debe primero identificar claramente sus necesidades financieras, luego tener muy claro cuáles son los parámetros básicos que se deben considerar para medir la conveniencia de un préstamo y, por último, determinar la capacidad de pagar la deuda contraída.

En cuanto a la dimensión factores externos, se aprecia en la tabla 2 según la opinión de los analistas y gerentes de los bancos universales del municipio Cabimas ciertas fortalezas dado una alta presencia de los factores: economía $(3,75)$, sector $(3,25)$ y actividad económica del cliente $(4,75)$ arrojando en promedio total una alta presencia $(3,92)$ de dichos factores.

Ello significa, según Sebastián y López (2005), un buen modelo de riesgo de crédito que cubre varios objetivos que a la vez se pueden utilizar debidamente para distintas aplicaciones existe congruencia con lo expuesto por Mascareña (2004), quien refiere que el estudio del crédito competente pretende no sólo proteger a los proveedores de posibles pérdidas, sino también proteger a los clientes desde la creación de más obligaciones de la deuda que no pueda ser resuelta de manera oportuna.

Tabla 2. Factores externos.

\begin{tabular}{lcc}
\hline \multicolumn{1}{c}{ Indicadores } & Media & Categoría \\
\hline Economía (E) & 3,75 & Alta presencia/ Fortaleza \\
Sector (S) & 3,25 & Moderada presencia/ Leve fortaleza \\
Actividad económica del cliente (AEC) & 4,75 & Muy alta presencia/ Fortaleza \\
\hline Dimensión & 3,92 & Alta presencia/ Fortaleza \\
\hline
\end{tabular}

En virtud de ello, se entiende que los factores externos representan una fortaleza para los bancos universales del municipio Cabimas, cuando el proceso de crédito se maneja de manera eficiente, y todos los involucrados se benefician de los esfuerzos., asimismo, el proveedor de este tiene una cantidad razonable de certeza de que el crédito proporcionado a un cliente se pagará dentro de los términos, o que regular los pagos mínimos serán recibidos en saldos de cuentas de crédito.

En síntesis, la tabla 3 presenta el resumen de los factores del riesgo identificándose una moderada presencia $(3,57)$ de los factores internos, y alta presencia $(3,92)$ de los factores externos, como una serie de consideraciones constituidos por una serie de políticas, límites y decisiones que se emplean para determinar el nivel de mezcla adecuada de riesgos que una institución que puede y debe asumir. 
Tabla 3. Factores del riesgo de créditos.

\begin{tabular}{lcc}
\hline \multicolumn{1}{c}{ Dimensión } & Media & Categoría \\
\hline Factores externos & 3,92 & Alta presencia/ Fortaleza \\
Factores internos & 3,31 & Moderada presencia/ Leve fortaleza \\
\hline \multicolumn{1}{c}{ Dimensión } & 3,57 & Alta presencia/ fortaleza \\
\hline
\end{tabular}

Las evidencias anteriores, tienen congruencia con los aportes Ahumada y Budnevich, (2001) y Salas y Saurina (2003), citados por Altuve y Hurtado (2018), para quienes existe un conjunto de características de la actividad bancaria que afectan la morosidad del sistema, entre ellas se puede mencionar, liquidez, rentabilidad, política crediticia, eficiencia en el manejo del riesgo. Además, de estos elementos de carácter microeconómico también existen factores macroeconómicos que explican el comportamiento y la tendencia de la morosidad del sistema.

No obstante, dado los resultados, existe fortaleza en el desarrollo de los factores del riesgo de créditos en los bancos universales del municipio Cabimas, para cuantificar y administrar el riesgo de crédito y reducir el riesgo de pérdida que pudiera resultar porque un cliente o contraparte incumpla con los términos convenidos.

Ahora bien, son relativamente comunes los modelos que explican los factores internos y externos que generan riesgo en el crédito y por ende que determinan la calidad futura de la cartera de créditos. Esto indica que el entorno económico donde la entidad bancaria se desenvuelve es tan importante como los factores relacionados con las políticas internas de conducción del banco.

Como complemento, una vez realizada la revisión bibliográfica sobre el riesgo en la cartera de microcréditos utilizados, se recolectaron los datos arrojados por el cuestionario aplicado se realizó un análisis exploratorio con la finalidad de determinar cuáles factores se encuentran más correlacionados.

En tal sentido, se procedió a calcular la matriz de correlación para determinar el impacto de cada una de los factores tanto interno como externos en los factores de riesgo en la cartera de microcréditos de los bancos universales. En la tabla 4 se presentan los resultados de la correlación simple para identificar el grado de asociación lineal entre los factores. Se observa que existe una correlación fuerte entre el riesgo legal y la estructura organizacional entre los factores internos, también se identifica una correlación fuerte entre la economía y la actividad económica del cliente. 
Tabla 4. Correlacion de los factores de riesgo.

\begin{tabular}{lrrrrrrr}
\hline & \multicolumn{1}{c}{ Política } & \multicolumn{1}{c}{ Riesgo O } & \multicolumn{1}{c}{ Riesgo L } & EO & Economía & Sector & AEC \\
\hline Política & 1 & & & & & & \\
Riesgo O & 0.05882353 & 1 & & & & & \\
Riesgo L & -0.1266601 & 0.6333005 & 1 & & & \\
EO & -0.41176471 & 0.7599606 & 0.76470588 & 1 & & \\
Economía & -0.6333005 & -0.3799803 & -0.45454545 & 0 & 1 & \\
Sector & -0.24253563 & -0.24253563 & 0.52223297 & 0 & -0.17407766 & 1 \\
AEC & 0.11764706 & -0.47058824 & $-2.8124 \mathrm{E}-17$ & -0.5 & -0.727606875 & $-2.8124 \mathrm{E} 17$ & 1 \\
\hline
\end{tabular}

En este sentido, se evidencio en relación a la estructura organizacional que quienes gestionan los microcréditos estrecha los vínculos de unión para permitir relaciones exitosas entre las partes involucradas y coordinan medidas destinadas a prevenir fraudes con documentos. Por tanto, en sus riesgos legales los bancos analizados cumplen con las disposiciones legales aplicables a las operaciones según la Superintendencia de bancos y se considera que las fallas operacionales son causadas por la usencia de leyes y reglamentos.

En relación con los factores externos, quienes gestionan los microcréditos aprovecha las oportunidades de crecimiento (periodo de inversión, rentabilidad del periodo, precios) que son activos de capital que añaden valor a la empresa, asimismo se considera que los indicadores económicos (tasas de interés, inflación) determinan un límite de crecimiento internamente sostenible.

Respecto a la actividad económica del cliente, para optar a un microcrédito el banco considera que mientras más datos se tengan sobre el solicitante menor será el grado de incertidumbre para adoptar la decisión, por otro lado opina que el otorgamiento del crédito está determinado por la capacidad de pago del solicitante que a su vez, está definida fundamentalmente por el flujo de solvencias y antecedentes crediticios.

Así, se estableció un modelo estadístico representado de la siguiente forma:

$$
\begin{gathered}
\mathrm{FRC}=\alpha_{1} \mathrm{RL}_{\mathrm{t}-1}+\alpha_{2} \mathrm{EO}_{\mathrm{t}-2}+\alpha_{3} \mathrm{RO}_{\mathrm{t}-1}+{ }_{\alpha 4} \mathrm{~S}_{\mathrm{t}-1}+\alpha_{5} \mathrm{AEC} C_{\mathrm{t}-1}-\alpha_{6} \mathrm{E}_{\mathrm{t}-1}-\alpha_{7} \mathrm{P}_{\mathrm{t}-1+\mu_{\mathrm{t}-1}} \\
\mathrm{FRC}=0,886968594+0,741467299+0,659082044+0,452910814+0,164770511-0,177393719- \\
0,164770511
\end{gathered}
$$

Donde FRC es la variable dependiente (factores de riesgo) del sistema bancario venezolano en el período $t$, mientras que del lado derecho de la ecuación se ubican las variables explicativas (factores internos y externos) con el signo esperado para cada una ellas, $\alpha$ representa la heterogeneidad no observable, es $\mu$ t el término de perturbación y t-1 representa los rezagos de cada una de las variables. 
En el modelo se muestra que las variables que mejor se ajustaron solo tienen un rezago. En cuanto a los signos esperados en las variables se tiene que las variables RL; EO; RO: $S$ y $A E C$ sin rezagos se rigen por los signos esperados teóricamente. Por su parte, las variables con un rezago presentaron signos contrarios a los esperados, ellas son E y $P$.

El análisis permite identificar una relación positiva entre el riesgo legal, la estructura organizacional, el riesgo operacional, sector, y la actividad económica del cliente como factores determinantes del riesgo de los microcréditos de las bancas analizadas, no obstante la economía y política tienen una relación negativa como factores externos que determinan los riesgos de crédito.

\section{CONCLUSIONES}

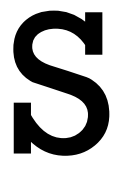

e logró determinar cada uno de los factores de riesgos involucrados en los entornos internos y externos del mercando bancario en el municipio Cabimas, revelando que el componente sistémico de los factores internos y externos seleccionados sobre los factores del riesgo en la cartera de microcréditos de los bancos universales puede ser de enorme importancia para el sistema bancario venezolano, dado que permite identificar cuáles son más significativos considerando el impacto de cada una de los factores del modelo.

\section{REFERENCIAS}

Ahumada, A., y Budnevich, C. (2001). Some measure of financial fragility in the Chilean banking system: an early warning indicator application. Banco Central de Chile, Documento de trabajo No $\quad 117 . \quad$ Disponible en: http://www.bcentral.cl/documents/20143/3201 9/bcch_archivo_168215_es.pdf/3d6d9ee3-d498a383-f1a1-c90b809e64e7

Altuve, J., y Hurtado, A. (2018). Análisis de los factores que influyen en la morosidad del sistema bancario venezolano (2005-2015) Revista Venezolana de Análisis de Coyuntura, vol. XXIV, N¹, 2018 Universidad Central de Venezuela

Gootkind, C. (2013). Alternative Asset Valuation and Fixed Income, Program Curriculum Volume 5
Level II 2013, CFA ${ }^{\circledR}$ Institute Ed. Boston: Pearson Custom Publishing, Reading 42

Mascareña, J. (2004). Medición y control de Riesgos Limusa. Tercera Edición. México D.F

Salas, V., y Saurina, J. (2003). Deregulation, market power and risk behavior in Spanish banks. European Economic Review, 47: 1061-1075

Sebastián, J., y López, K. (2005). Análisis contable de la rentabilidad empresarial. Centro de estudios financieros. Madrid

Soley, J., y Rahnema, A. (2006). Harvard Deusto Finanzas y Contabilidad, ISSN 1134-0827, No. 64, 2005, pp. 40-49

Tamayo, M. (2009). El Proceso de la investigación científica. Quinta edición. Editorial Limusa. México 